\title{
Application of a Fast Boundary Element Algorithm for Open Floating Potential Electrostatic Problem
}

\author{
Deng Jun, Li Licheng, Hao Yanpeng, Chen He, Wang Jun, and Zheng Xin
}

\begin{abstract}
A fast boundary element method (BEM) are presented for the analysis of open floating potential electrostatic problem, where preconditioners base on adjacent nodes or leaves can improve the ill-conditioned coefficient matrix implicitly formed by BEM. Numerical results clearly demonstrate that preconditioners can improve computation time and storage in solving electrical field for site $\mathrm{AC}$ withstand power current transformer (CT), the max relative error of this algorithm is $9.5 \%$ in comparison to CDEGS, semi-analytical computation method, non-precondition and the finite element. Application of this algorithm can available technique to determinate the minimum distance of site $\mathrm{AC}$ withstand power from the threshold value of ICNIRP, optimist corona ring design and solve other open floating potential electrostatic problem in power system.
\end{abstract}

Index Terms-Boundary element methods, electric fields, substations, power transmission and transformation system.

\section{INTRODUCTION}

Many advantages over conventional ac can be obtained by high-voltage (HV) DC and AC for bulk-power and long-distance power transmission. Consequently, several $\pm 500 \mathrm{kV}$ HVDC and HVAC transmission lines have been built in China, and the EHV projects of $\pm 800 \mathrm{kV}$ DC and $\pm 1000 \mathrm{kV}$ AC have been developed for the energy needs. However, the further research associated with $\mathrm{HV}$ transmission technology is being studied for the stable operation of power devices and system. Especially, the numeric algorithm should be presented for the solution of open floating potential problem in a heterogeneous dielectric medium consisting of several regions with different permittivity within which there are several known conductors, which are widely distributed in the primary equipments of power transmission and transformation system.

Many research works have focused on analysis of floating potential problems. For example, a simple charge simulation method has been employed to calculate electric fields for composite dielectric arrangement with a floating conductor [1]. However, the position and quantity of the simulation charge are difficult to decide for the application. Furthermore, the approach of using analytical-numerical

Manuscript received March 3, 2013; revised July 2, 2013.

Deng Jun is with Maintenance \& Test Center, EHV, China Southern Power Grid, Guangzhou Guangdong 510663, China and School of Electric Power, South China University of Technology, Guangzhou Guangdong 510640, China (e-mail: reacherempire@163.com).

Hao Yanpeng and Li Licheng are with School of Electric Power, South China University of Technology

Chen He, Wang Jun and Zheng xin are with Maintenance \& Test Center, EHV, China Southern Power Grid. method to simulate 3-dimensional electric fields in several dielectrics with floating potential is adopted while it can't be presented for complex boundary problem [2]. In addition, the finite element method and fictitious domain method on floating potential model has been carried out [3]. Therefore, the effective algorithm should be developed for the analysis of floating potential problem existing in $\mathrm{HV}$ power transmission and transformation system with the characteristic of large scale and complex boundary.

Using the fast multipole (FM) to solve floating potential problems with boundary element method (BEM) reduces the computational cost and the memory requirements. BEM has enjoyed the reputation in handling complicated geometries and infinite domains, and FMM can be used to accelerate matrix-vector multiplications in the BEM iterative process [4]. However, the convergence of iterative methods is substantially slowed down due to the condition number of the coefficient matrix resulted from BEM and FMM, precondition can be employed to modify coefficient matrix and converge to exact solution in significantly fewer iteration steps.

Preconditioners technology has been developed for the improvement of coefficient matrix. Preconditioner based on the block diagonal matrix corresponding to the non-interacting particles for 3D many particle problems is found to be fewer iteration numbers [5]. a preconditioner is well suited for fast multipole BEM to the analysis of 3D crack problems [6]. The preconditioning of fast multipole BEM has been solved successfully by a new technique based on the inverse of the block diagonal matrix corresponding to element or node neighbors with satisfactory rate of convergence in modeling electron guns [7]. Remarkable results have been obtained from the 3-D BEM analysis of electron guns problem solved by sparse inverse preconditioner based on the leaves [8].

In this paper, the detailed theory principle for the floating potential problems solved by the fat BEM algorithm has been illustrated, preconditioners based on adjacent nodes and leaves have been adopted to improve the iterative convergence rate. The behavior of accuracy, computational cost and application performance of the preconditioner fast BEM for the simulation of the floating potential problems are clearly demonstrated by the numeric results.

\section{Formulation OF OPEn FloAting Potential Electrostatic Problem}

The math model of the open floating potential electrostatic problems for $\mathrm{HV}$ power transmission and transformation system containing $\mathrm{n}$ closed insulation dielectric regions with 
known permittivity and $\mathrm{m}$ floating conductors with known or unknown potential is shown Fig.1, where the dielectric regions and conductors are enclosed in a common open air region $\Omega$ extending to infinity. Furthermore, the conductors and insulation dielectric can be denoted by $\mathrm{c} 1, \mathrm{c} 2, \ldots \mathrm{cm}$ and $\mathrm{d} 1, \mathrm{~d} 2, \ldots \mathrm{dn}$, respectively. Moreover, let $\Gamma_{c 1}, \Gamma_{c 2}, \Gamma_{c m}$ and $\Gamma_{d 1}, \Gamma_{d 2}, \Gamma_{d m}$ denote the interfaces of the conductors and insulation dielectric with the air, respectively. In addition, $S_{1}$ and $S_{2}$ are the boundary with the given electric potential $u$ and the normal of the dielectric displacement $\psi$, respectively. Consequently, the floating potential problem can be simulated by the following governing equations.



Fig. 1. Model containing floating conductors and multiple dielectrics.

$$
\begin{aligned}
& \left.\nabla^{2} \varphi\right|_{\Omega}=0 \\
& \left.\varphi\right|_{S_{1}}=u \\
& \left.\frac{\partial \varphi}{\partial n}\right|_{S_{2}}=\psi \\
& \left.\varepsilon_{a} \frac{\partial \varphi_{a}}{\partial n}\right|_{\Gamma_{d i}}=\left.\varepsilon_{d i} \frac{\partial \varphi_{d i}}{\partial n}\right|_{\Gamma_{d i}} \quad(i=1,2 \bullet \bullet m) \\
& \left.\oint \varepsilon_{a} \frac{\partial \varphi_{a}}{\partial n}\right|_{\Gamma_{c j}}=\left.q_{c j} u\right|_{\Gamma_{c j}}=u_{c j} \quad(j=1,2 \bullet \bullet n)
\end{aligned}
$$

where $\varphi$ is the electric potential of the calculation domain $\Omega$. $\varepsilon_{a}$ and $\varepsilon_{d i}$ are the relative permittivity of air and insulation dielectric, respectively. $\varphi_{a}$ and $\varphi_{d i}$ are the interface potential of air and insulation dielectric, respectively. $u_{c j}$ is the given potential on the surface of conductors. $q_{c j}$ is the total charge of the floating conductor $\boldsymbol{c j}$.

The well-known boundary integration equation based on the fundamental solution for 3D Laplace equation can be employed to represent the electric potential $\varphi$ by using the following equation (2).

$$
\sum_{j=1}^{N} H_{i j} \phi_{j}+\sum_{j=1}^{N} G_{i j} Q_{j}=0 \quad(i=1,2 \bullet \bullet N)
$$

where $N$ is the number of the discrete elements. $H_{i j}$ and $G_{i j}$ are the coefficients for boundary element method (BEM) expressed as

$$
G_{i j}=\oint_{\Delta S_{j}} \frac{1}{4 \pi \varepsilon_{0} r} d s
$$

$$
H_{i j}= \begin{cases}\oint_{\Delta S_{j}} \frac{1}{4 \pi \varepsilon_{0}} \frac{\partial\left(\frac{1}{r}\right)}{\partial n} d s & i \neq j \\ \oint_{\Delta S_{j}} \frac{1}{4 \pi \varepsilon_{0}} \frac{\partial\left(\frac{1}{r}\right)}{\partial n} d s-\frac{1}{2} & i=j\end{cases}
$$

where $\varepsilon_{0}$ is the air permittivity, $\mathrm{r}$ denote the distance between the resource point and the field point, $\Delta S_{j}$ is the surface element of conductors or insulation dielectric.

Substituting (2) into (1) yields a set of linear algebraic equations for all nodes on the boundary of the problem domain.

$$
\begin{gathered}
{\left[\begin{array}{llll}
H_{S_{1}} & H_{S_{2}} & H_{C} & H_{d}
\end{array}\right]\left[\begin{array}{l}
\phi_{S_{1}}\left(N_{1}\right) \\
\phi_{S_{2}}\left(N_{2}\right) \\
\phi_{C}\left(N_{3}\right) \\
\phi_{d}\left(N_{4}\right)
\end{array}\right]} \\
+\left[\begin{array}{llll}
G_{S_{1}} & G_{S_{2}} & G_{C} & G_{d}
\end{array}\right]\left[\begin{array}{l}
Q_{S_{1}}\left(N_{1}\right) \\
Q_{S_{2}}\left(N_{2}\right) \\
Q_{C}\left(N_{3}\right) \\
Q_{d}\left(N_{4}\right)
\end{array}\right]=0
\end{gathered}
$$

where $N_{1}$, and $N_{2}$ denote the number of discrete elements from the surface of $S_{1}$ and $S_{2}$, respectively. $N_{3}$ and $N_{4}$ denote the number of discrete elements from the interfaces of the conductors and insulation dielectric with the air.

It seems that equation (5) can't be determined by the unique solution when there are $N_{3}+m$ unknowns. Therefore, the coupling equation (6) should be added according to the surface continuity condition for floating conductors and insulation dielectric.

$$
\begin{aligned}
& {\left[\begin{array}{ccc}
\varepsilon_{a} G_{d j}^{a} & -\varepsilon_{d j} G_{d j}^{d} & 0 \\
0 & 0 & \varepsilon_{a} G_{C}
\end{array}\right]\left[\begin{array}{l}
Q_{a}\left(N_{4}\right) \\
Q_{d}\left(N_{4}\right) \\
Q_{C}(m)
\end{array}\right]=\left[\begin{array}{c}
0 \\
q_{C}
\end{array}\right]} \\
& q_{C}=\left[\begin{array}{llll}
q_{c 1} & q_{c 2} & \bullet \bullet & q_{c m}
\end{array}\right]^{T}
\end{aligned}
$$

where $G_{d j}^{a}$ and $G_{d j}^{d}$ are potential coefficient computed from the boundary of air and insulation dielectric, respectively. $Q_{a}$ and $Q_{d}$ are the normal of the dielectric displacement derived from the boundary of air and insulation dielectric, respectively. $G_{C}$ and $Q_{C}$ are the potential coefficient and the normal of the dielectric displacement derived from the boundary of air and floating conductors, respectively.

The fast multipole (FM) can accelerate matrix-vector multiplications, which can be used to an iterative solver for the solution of the linear equation (5) and (6). And the memory requirement can be reduced without computing coefficient matrix. The FM implement for equation (5) and (6) can be represented by the application of superposition to near and far elements potential contribution, where near and far elements potential contribution can be computed by conventional direct evaluation of the integrals equation (2) and FM, respectively. Consequently, the matrix-vector multiplications in equation (5) and (6) can be rewritten as 


$$
\begin{aligned}
& {\left[\begin{array}{llllll}
H_{S_{1}} & H_{S_{2}} & H_{C} & H_{d} & \varepsilon_{a} G_{d j}^{a} & \varepsilon_{a} G_{C}
\end{array}\right]\left[\begin{array}{c}
\phi_{S_{1}}\left(N_{1}\right) \\
\phi_{S_{2}}\left(N_{2}\right) \\
\phi_{C}\left(N_{3}\right) \\
\phi_{d}\left(N_{4}\right) \\
Q_{a}\left(N_{4}\right) \\
Q_{C}(m)
\end{array}\right]} \\
& +\left[\begin{array}{llllll}
G_{S_{1}} & G_{S_{2}} & G_{C} & G_{d} & -\varepsilon_{d j} G_{d j}^{d} & 0
\end{array}\right]\left[\begin{array}{c}
Q_{S_{1}}\left(N_{1}\right) \\
Q_{S_{2}}\left(N_{2}\right) \\
Q_{C}\left(N_{3}\right) \\
Q_{d}\left(N_{4}\right) \\
Q_{d}\left(N_{4}\right) \\
Q_{C}(m)
\end{array}\right] \\
& =\sum_{q}^{N_{\text {naer }}} G_{i j} Q_{j}+\sum_{q}^{N_{\text {maer }}} H_{i j} \phi_{j}+ \\
& \sum_{q}^{N_{\text {far }}} \frac{1}{4 \pi} \sum_{n=0}^{\infty} \sum_{m=-n}^{n} R_{n}^{m} \times L_{n}^{m}
\end{aligned}
$$

where $N_{\text {near }}$ and $N_{\text {far }}$ are the total number of near and far elements, respectively. $R_{n}^{m}$ and $L_{n}^{m}$ can be found in Ref [9].

\section{NUMERICAL IMPLEMENTATION}

With the increase of the media quantity and model scale, the condition number of the coefficient matrix defined by $\mathrm{A}$ resulted from direct evaluation in equation (7) significantly deteriorates. Therefore, preconditioner technique based on adjacent nodes and levels can be employed to obtain higher rate of convergence when the characteristic of FM is taken into account. Matrix $A^{*}$ can be approximated as original matrix $A$ by dropping elements of $A$ below a prescribed threshold, where $A^{*}$ can be obtained by the sparse inverse algorithm based on the minimization of Frobenius norm as follow and matrix $M$ is determined to approximate the inverse of $A^{*}$.

$$
M \bullet A^{*} \approx I \rightarrow \min \left\|I-A^{* T} \bullet M^{T}\right\|
$$

where $I$ is the identity diagonal matrix.

\section{A. Preconditioner Based on Adjacent Nodes}

Two nodes are considered to be far enough from each other when they are not in the same six-noded triangular element. Consequently, the interaction related to these two nodes are zeros in coefficient matrix $A$. the principle described above as preconditioner based on adjacent nodes can be presented to rewrite equation (8) as

$$
\left\|I-A^{* T} \bullet M^{T}\right\|^{2}=\sum_{j=1}^{N}\left\|e_{j}-A^{* T} \bullet m_{j}\right\|^{2}
$$

where $e_{j}$ and $m_{j}$ denotes the $j$ th column of $I$ and $M^{T}$, respectively. The computation of $M^{T}$ is reduced to solutions of $N$ independent linear least-squares problems. The algorithm to calculate every column $m_{j}$ of $M$ is the solution of a linear system in Ref [7].

\section{B. Preconditioner Based on Leaves}

Two nodes are not a neighbor while they belong to different leaves cubes, which are the basic elements to implement the FM algorithm. Preconditioner based on leaves can zero out the direct evaluation related to these two nodes in coefficient matrix $\boldsymbol{A}$. The corresponding algorithm is given [8]

$$
M_{m_{n} \times m_{n}}^{n} \cdot A_{m_{n} \times m_{n}}^{n}=I_{m_{n} \times m_{n}}^{n} \rightarrow M_{m_{n} \times m_{n}}^{n}=\left[M_{m_{n} \times m_{n}}^{n}\right]^{-1}
$$

where $n$ and $m_{n}$ denotes index of a leaf cube and the order of sub-matrix $\left(A^{n}\right)\left(m_{n \times} m_{n)}\right.$ related to the $n$th leaf, respectively. $\left(A^{n}\right), m_{n \times} m_{n)}$ denote the $n$th diagonal block of $\left(A^{*}\right)$, formed by interactions of all nodes in this leaf. $\left(M^{n}\right)_{(} m_{n \times} m_{n)}$ and $I$ $\left(m_{n \times} m_{n)}\right.$ denotes the $n$th diagonal block of $M$ and the identity matrix of order $m_{n}$, respectively.

\section{Solver of Bi-Conjugate Gradient Method}

The bi-conjugate gradient method, which approaches great advantage in smaller storage requirement by replacing the orthogonal sequence of residuals by two mutually orthogonal sequences, is an effective method for equation (7). The implement steps can be described below.

1) The initial solution vector for equation (7) can be set to $x_{0}$, then the error can be defined as $r_{0}=A x_{0}-b$.

2) Two orthogonal sequences can be obtained by the following procedure.

$$
\begin{aligned}
& \text { for } k=0,1,2 \bullet \bullet \\
& \text { solve } z_{k} \text { from } M z_{k}=r_{k} ; \\
& \text { solve } z_{k}^{*} \text { from } M^{T} z_{k}^{*}=r_{k}^{*} ; \\
& \text { if } k=0 \\
& p_{k+1}=z_{k} ; p_{k+1}^{*}=z_{k}^{*} \\
& \text { else } \\
& \beta_{k}=\left(z_{k}, r_{k}^{*}\right) /\left(z_{k-1}, r_{k-1}^{*}\right) \\
& p_{k+1}=z_{k}+\beta_{k} p_{k} ; p_{k+1}^{*}=z_{k}^{*}+\beta_{k} p_{k}^{*} \\
& \text { end }
\end{aligned}
$$

3) The solution and error for equation (7) can be given as

$$
\begin{aligned}
& \alpha_{k+1}=\left(z_{k}, r_{k}^{*}\right) /\left(p_{k+1}^{*}, A p_{k+1}\right) \\
& x_{k+1}=x_{k}+\alpha_{k+1} p_{k+1} \\
& r_{k+1}=r_{k}-\alpha_{k+1} A p_{k+1} ; r_{k+1}^{*}=r_{k}^{*}-\alpha_{k+1} A^{T} p_{k+1}^{*}
\end{aligned}
$$

4) If the error $r_{k+1}$ satisfies the computation accuracy, the vector $x_{k+1}$ can be as the final solution and exit the procedure. Otherwise, $r_{k+1}$ and $x_{k+1}$ should be substituted into step (2) until $r_{k+1}$ has achieved the given accuracy.

\section{NUMERICAL RESUlTS}

In this section, the results of the numerical analysis showing the behavior of calculation efficiency, accuracy and application performance from different engineering examples are presented.

\section{A. A Fast Boundary Element Algorithm for Site Ac Withstand Power Ct}

The calculation efficiency performance and accuracy of proposed numerical technique is demonstrated with the electrical field distribution for site AC withstand power CT when bus-bar and outgoing line related equipments are 
charged operation in a $500 \mathrm{KV}$ outdoor substation, whose general view and essential dimensions are shown in Fig. 2.

Eigenvalue of non-precondition, preconditioner based on adjacent nodes and preconditioner based on leaves for the fast BEM are showed in Fig. 3, Fig. 4 and Fig. 5, respectively. It can be seen that, most eigenvalues of the non-precondition fast BEM are clustered around 0 , while eigenvalues of the preconditioned fast BEM are well clustered around 1, either preconditioner based on adjacent nodes or leaves. Therefore, the preconditioned fast BEM can result in rapid convergence because of its matrix condition number is lower.

The three curves in Fig. 6 are related to non-precondition, preconditioner based on adjacent nodes and preconditioner based on leaves for the fast BEM, respectively. It appears that the preconditioner fast BEM need less iteration steps than non-precondition for the same accuracy.

Fig. 7 shows the dependence of iteration step number on the maximum number of elements in leaves, it can be seen that the preconditioner fast BEM need less iteration steps than non-precondition when the elements number in leaves is the same. In addition, all the curves are oscillatory; this phenomenon is related to shape change of the tree structure with the elements number in leaves.

Fig. 8 and Fig. 9 show the dependence of memory and calculation time on the maximum number of elements in leaves, respectively. It can be observed that preconditioner based on adjacent nodes or leaves is much faster and less memory than non-precondition in computational time and storage of algorithm. And less memory can be required for preconditioner based on leaves than that on adjacent nodes, the reason is that the matrix for leaves is smaller than that of nodes.
Fig. 10 shows the relative error for this problem using both CDEGS [10], semi-analytical computation method [2], non-precondition, preconditioner based on adjacent nodes and leaves of the fast BEM on the plane of $X=-5.5$ and $Z=1.5$. As we can see that both the preconditioner fast BEM don't influence the accuracy of numerical result significantly. The max relative error is $6 \%$ accepted by engineer numerical calculation in comparison to CDEGS, semi-analytical computation method and non-precondition.

Fig. 11 show the contour plot for the results of electrical field distribution for site AC withstand power CT on the plane of $X=-5.5$ for this problem using the proposed algorithm, where the control plane has been operated by test staff. It can be seen that electric field strength does not exceed $10 \mathrm{KV} / \mathrm{m}$ from Fig. 2 when the plane height $\mathrm{Y}=1.5$ is corresponding to the human head, where $10 \mathrm{KV} / \mathrm{m}$ is the threshold value in occupational exposure according to the regulation of ICNIRP.

The electric field on the head of operation staff versus different distance can be seen from Fig. 12, where the head of operation staff is corresponding to the plane of $\mathrm{Z}=1.8 \mathrm{~m}$ and the distance is defined as the modulus value of the displacement from the head of operation staff for site AC withstand equipments console to the sag point of the connecting line between the $\mathrm{B}$ and $\mathrm{C}$ phase $\mathrm{CT}$. the minimum distance is $8.6 \mathrm{~m}$ according to the threshold value of ICNIRP when the height of CT is $8.8 \mathrm{~m}$. However, the $5 \mathrm{~m}$ distance to guarantee staff life for $500 \mathrm{kV}$ AC equipments is less than that of Ref [11]. Therefore, the reasonable HV primary test equipments can be located by the application of this algorithm for the staff exposure below $10 \mathrm{kV} / \mathrm{m}$.

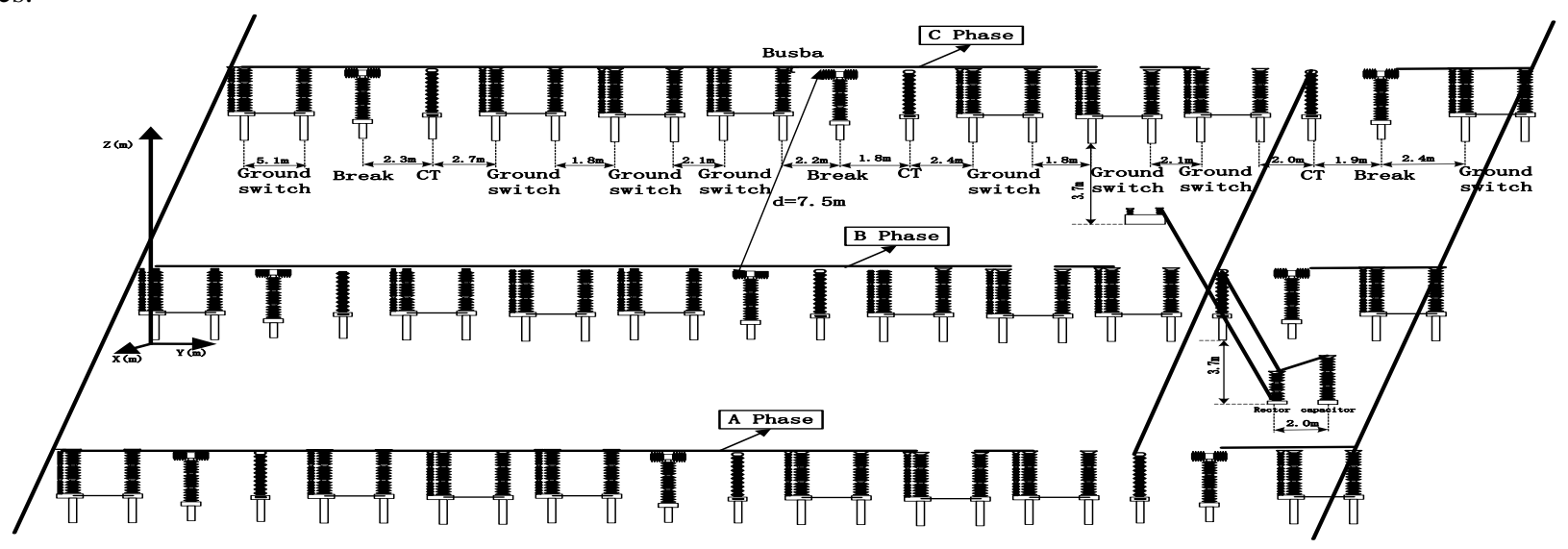

Fig. 2. Equipments distribution for site $\mathrm{AC}$ withstand power $\mathrm{CT}$ in a $500 \mathrm{KV}$ outdoor substation.

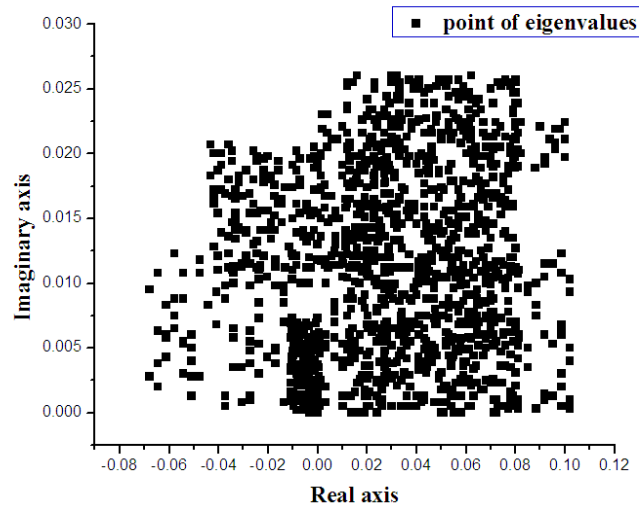

Fig. 3. Eigenvalue distributions of non-precondition.

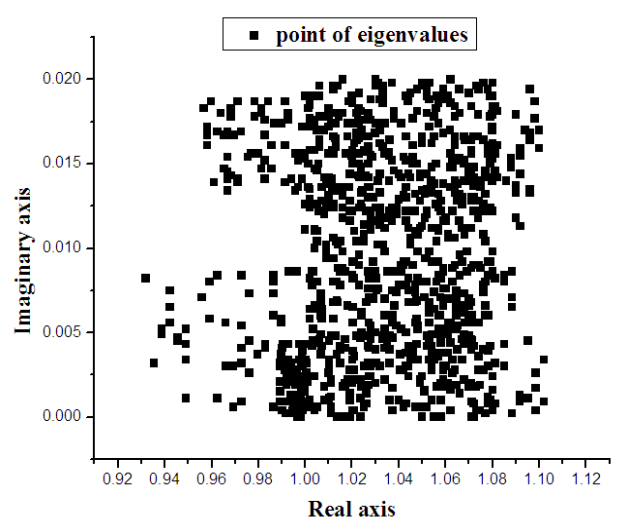

Fig. 4. Eigenvalue distributions of preconditioner based on adjacent nodes. 


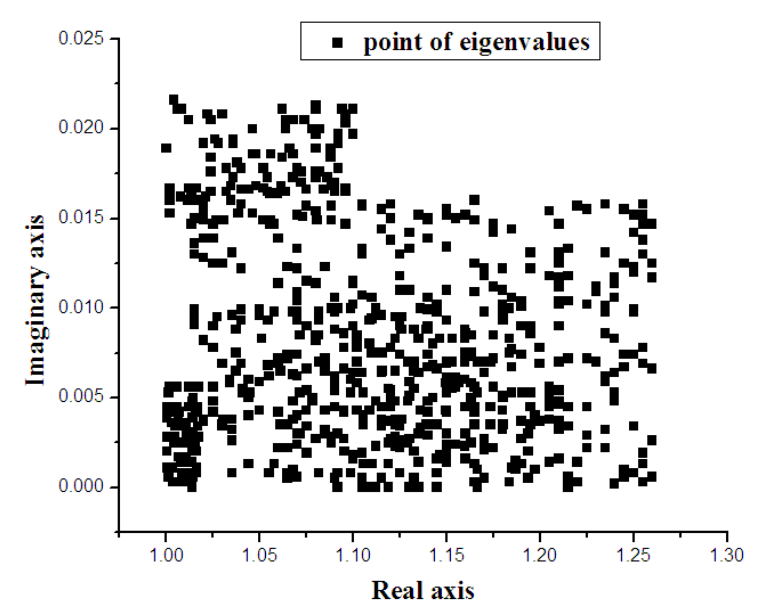

Fig. 5. Eigenvalue distributions of preconditioner based on leaves.

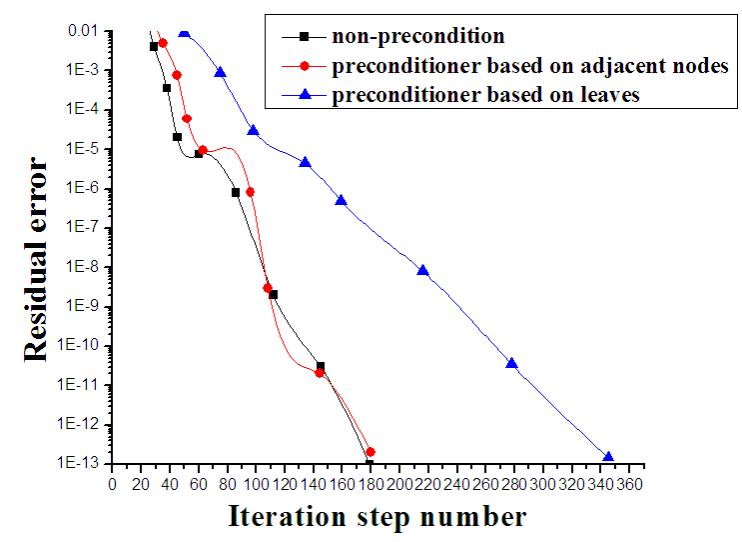

Fig. 6. Residual error vs. iteration number.

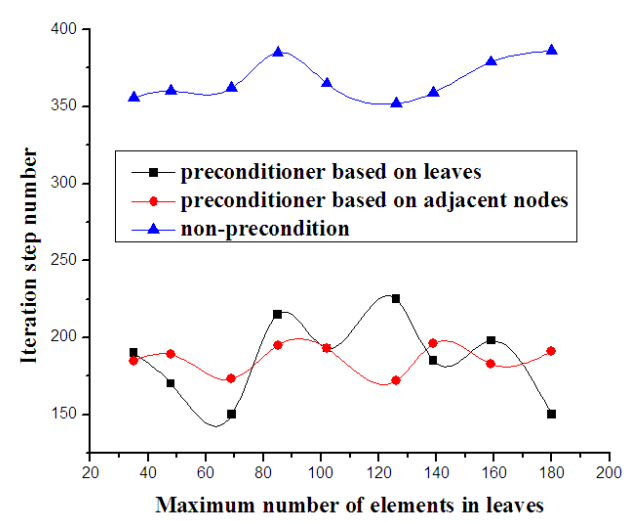

Fig. 7. Iteration step number vs. maximum number of elements in leaves.

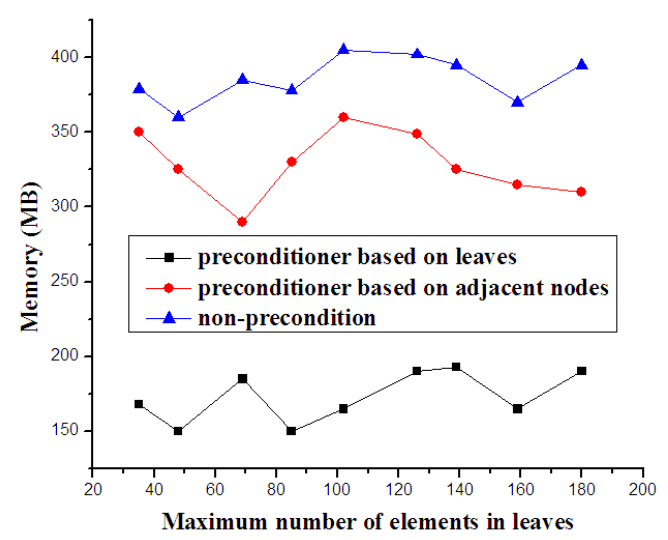

Fig. 8. Memory vs. maximum number of elements in leaves.

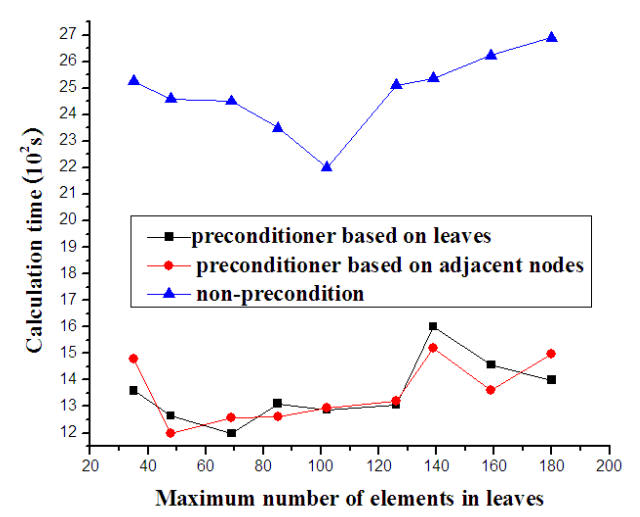

Fig. 9. Computation time vs. maximum number of elements in leaves.

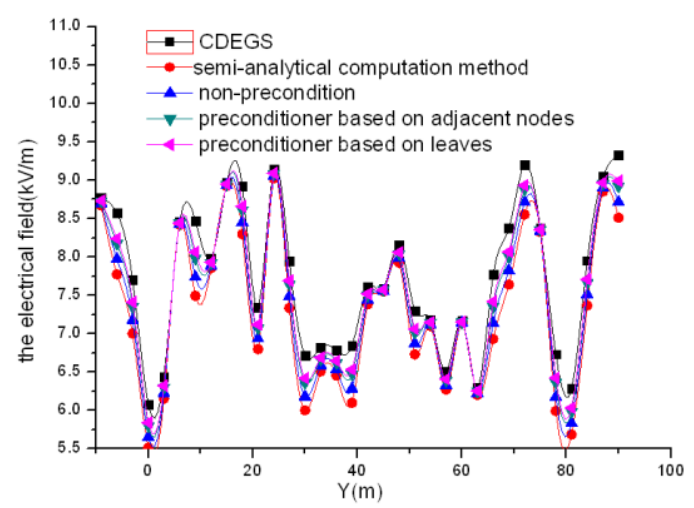

Fig. 10. The electrical field calculation from CDEGS , semi-analytical computation method, non-precondition, preconditioner based on adjacent nodes and leaves of FMM BEM on the plane of $\mathrm{X}=-5.5$ and $\mathrm{Z}=1.5$.

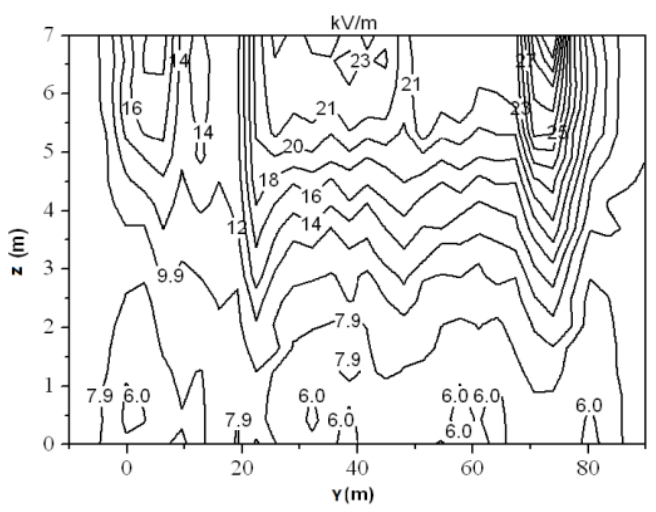

Fig. 11. electric field of on the plane of $Y=-5.5$.

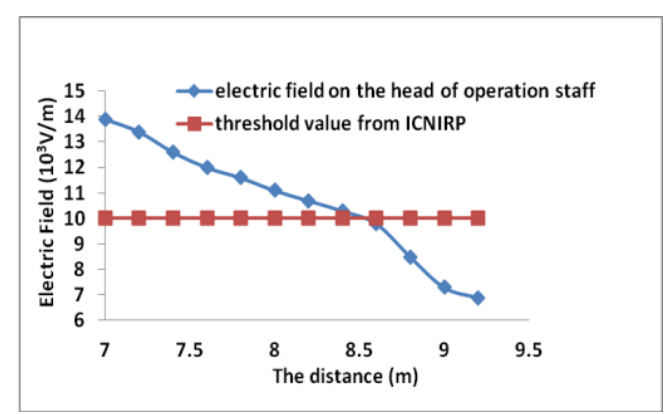

Fig. 12. The electric field vs. the distance.

B. A Fast Boundary Element Algorithm for $\pm 800 \mathrm{kv}$ Uhv Dc Corona Ring Optimization Design

This fast boundary element algorithm can be employed to optimize design the $\pm 800 \mathrm{kV}$ UHV DC corona ring when The calculation accuracy can also be compared with the finite element method. 
The model for $\pm 800 \mathrm{kV}$ UHV DC corona ring is described as below. The total height of the tower for hanging the insulators, corona rings, conductor and ground wire is $50 \mathrm{~m}$, where the height and length for insulators are $40 \mathrm{~m}$ and $8.75 \mathrm{~m}$, respectively. Furthermore, the distance for bipolar conductor and ground wire are $22 \mathrm{~m}$ and $20 \mathrm{~m}$, respectively. Moreover, the insulator structure contains four small sheds of diameter $0.098 \mathrm{~m}$, one middle shed of diameter $0.162 \mathrm{~m}$, one big shed of diameter $0.24 \mathrm{~m}$. The type of the bipolar conductors is $6 \times$ ACSR-630/45 and its bundled radius is $0.45 \mathrm{~m}$.

The accuracy comparison of the fast boundary element algorithm and the finite element method can be presented in Fig. 13. All methods were adjusted to obtain the positive pole insulator axial electric field distribution with the same relative accuracy10 6 . The range of the relative error between these two algorithm is from 5\% 9.5\%, which can be accepted by engineering design and computation. Obvious gradient trend of electric field can be found the same along the positive pole insulator axial distance. The maximum electric field for the fast boundary element algorithm and the finite element method are $8.5 \times 105 \mathrm{~V} / \mathrm{m}$ and $9.3 \times 105 \mathrm{~V} / \mathrm{m}$ on the corona ring surface.

The effect of height $h$, the inner diameter $r$ and outter diameter $\mathrm{R}$ for the corona rings can be depicted on the highest electric field at the surface of HV fittings, corona ring and insulator sheath in Fig. 14, Fig. 15 and Fig. 16. the more higher electric field on the surface of HV fittings can be obtained with increasing the outter diameter or decreasing the inner diameter and the height of corona. Furthermore, the lower electric field on the surface of $\mathrm{HV}$ insulator sheath can also be achieved as the outter and inner diameter increases. However, the effect of height $\mathrm{h}$ on electric field on the surface of HV insulator sheath can be neglected resulting from its litter influence. Moreover, the electric field on the surface of $\mathrm{HV}$ corona ring decreases with the grow of the height, the inner diameter and outter diameter.

The optimal dimension for corona ring are $R=325 \mathrm{~mm}$, $r=75 \mathrm{~mm}$ and $h=110 \mathrm{~mm}$ according to the variety of geometry parameter. Therefore, the maximum electric field for above size on surface of fittings, insulator sheath and corona ring are $8.1 \times 105 \mathrm{~V} / \mathrm{m}, \quad 6.5 \times 105 \mathrm{~V} / \mathrm{m}$ and $10.1 \times 105 \mathrm{~V} / \mathrm{m}$, the potential contour distribution on the sysmetry plane of the model can be seen in Fig. 17.

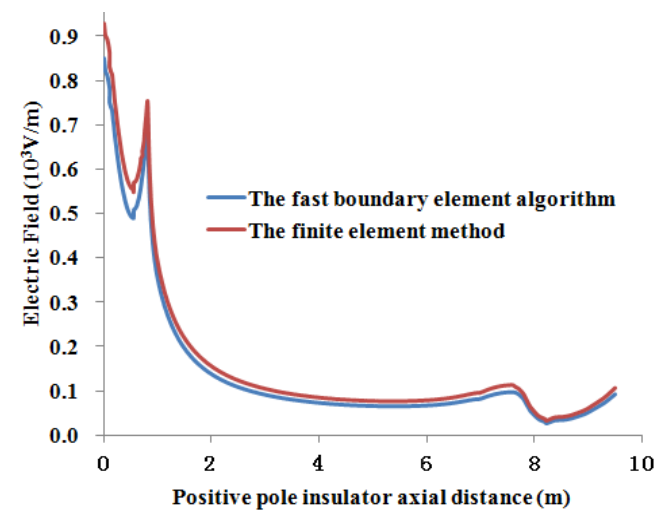

Fig. 13. The accuracy comparison of the fast boundary element algorithm and the finite element method.

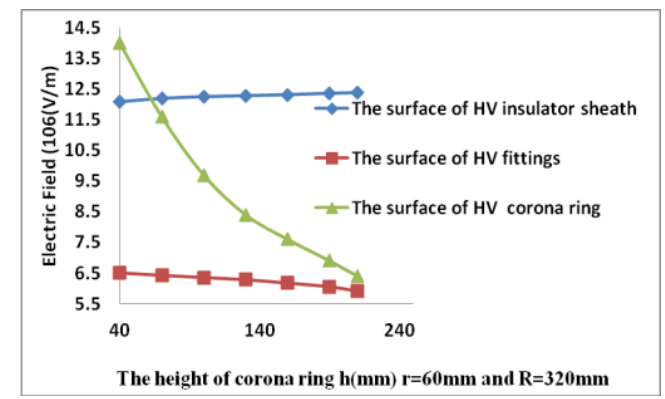

Fig. 14. Effect of geometry height of corona rings on the highest electric field for different surface.



Fig. 15. Effect of the inner diameter of corona rings on the highest electric field for different surface.

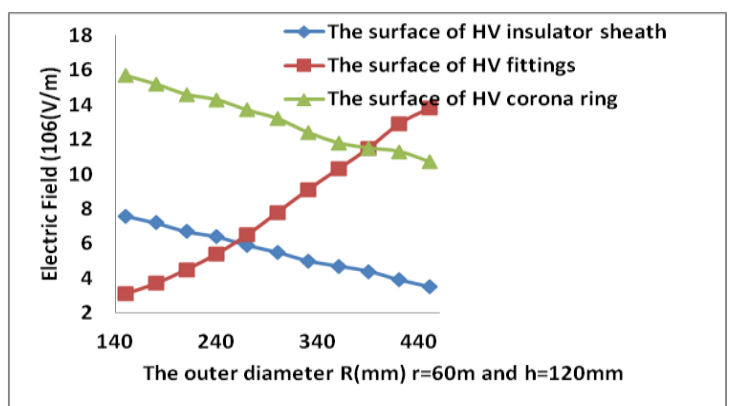

Fig. 16. Effect of the outer diameter of corona rings on the highest electric field for different surface.

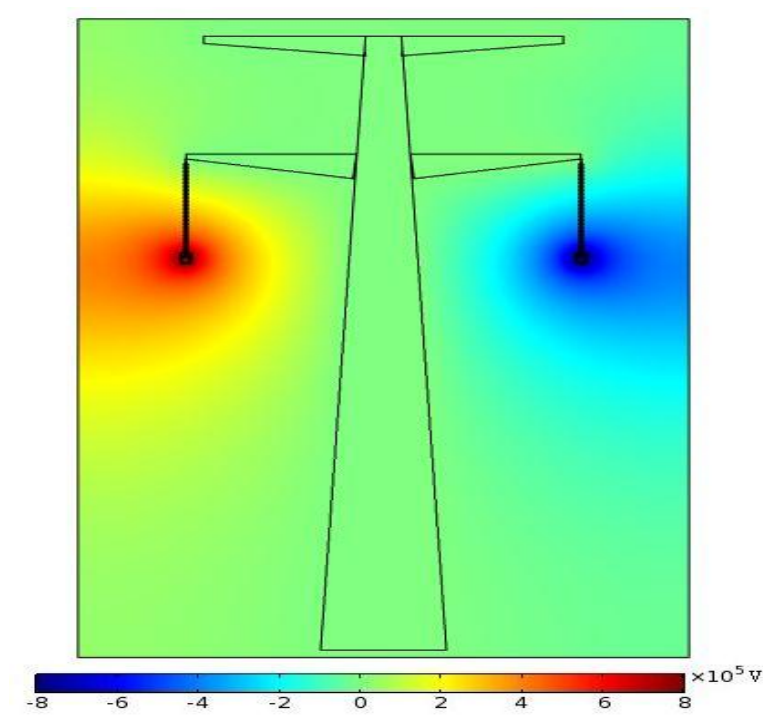

Fig. 17. The potential contour distribution on the sysmetry plane of the model.

\section{CONCLUSION}

The behavior of calculation efficiency, accuracy and application performance for the fast boundary element algorithm are investigated for site AC withstand power CT and $\pm 800 \mathrm{kV}$ UHV DC corona ring optimization design. the 
main conclusions from these investigations can be summarized as follows:

1) Preconditioner based on adjacent nodes or leaves can result in rapid convergence due to its matrix condition number is lower, where eigenvalues for preconditioner are well clustered around 1 .

2) The max relative error of this algorithm is $6 \%$ in comparison to CDEGS, semi-analytical computation method and non-precondition, while The range of the relative error between these two algorithm is from $5 \% \sim 9.5 \%$ compared with the finite element. Both of above error can be accepted by engineering design and computation.

3) Preconditioner based on adjacent nodes or leaves is much faster and less memory than non-precondition in computational time and storage of algorithm from the application of site AC withstand power CT.

4) The minimum distance is $8.6 \mathrm{~m}$ according to the threshold value of ICNIRP when the height of CT is $8.8 \mathrm{~m}$, where $8.6 \mathrm{~m}$ is larger than $5 \mathrm{~m}$ from safety code of electric power industry for guaranteeing staff life.

5) The optimal dimension for corona ring are $R=325 \mathrm{~mm}$, $r=75 \mathrm{~mm}$ and $h=110 \mathrm{~mm}$ according to the variety of geometry parameter. The fast boundary element method can be practical technique for open floating potential electrostatic problem in power transmission and transformation system.

\section{ACKNOWLEDGMENT}

This work is supported by the National High technology Research and Development of 863 programs (2010AA0522555001).

\section{REFERENCES}

[1] N. O. Morales and E. S. Asenjo, "Calculation of 3-dimensional fields in a medium with several dielectrics," IEEE Transactions on Dielectrics and Electrical Insulation, vol. 4, no. 2, April, 1997.

[2] N. O. Morales, E. S. Asenjo, and J. L. Inostroza, "A generalized analytical-numerical method for the calculation of 3-dimensional $\mathrm{h}$. $\mathrm{v}$. fields," IEEE Trans. on Electrical Insulation, vol. 23, pp. 929-936, 1988.

[3] A. Konrad and M. Graovac, "The finite element modeling of conductors and floating potentials," IEEE Transactions on Magnetics, vol. 32, no. 5, September, 1996.

[4] V. Rokhlin, "Rapid solution of integral equations of classical potential theory," J. Comput Phys, vol. 60, pp. 187-207, 1985.

[5] Y. H. Fu, K. J. Klimkowski, G. J. Rodin, E. Berger, J. C. Browne, J. K. Singer, and R. A. Geijn, “A fast solution method for three-dimensional many-particle problems of linear elasticity," Int J Numer Meth Eng; vol. 42, pp. 1215-29, 1998

[6] K. Yoshida, N. Nishimura, and S. Kobayashi, "Application of new fast multipole boundary integral equation method to crack problems in 3D," Eng. Anal Boundary Elements, vol. 25, pp. 239-47, 2001.

[7] M. Benzi, "Preconditioning techniques for large linear systems: a survey," J. Comput Phys, vol. 182, pp. 418-77, 2002.

[8] T. Nishida and K. Hayami, "Application of the fast multipole method to the 3-D BEM analysis of electron guns," in Boundary elements XIX. Southampton, M. Marchetti et al., eds., NY: Computational Mechanics, pp. 613-22, 1997.

[9] H. Wang and Z. H. Yao, "A new fast multipole boundary elementmethod for large scale analysis of mechanical properties in 3D particle-reinforced composites," Comput Model Eng Sci, vol. 7, pp. 85.

[10] S. Nikolovski and P. Mari, "Electromagnetic field calculation of transformer station $400 / 110 \mathrm{kv}$ ernestinovo using the cdegs software," Journal of Electrical Engineering, vol. 58, no. 4, 207-213, 2007.

[11] GB 26860-2011, Safety code of electric power industry-Electric part of power plants and transformer substations, SAC/TC 226, 2011.

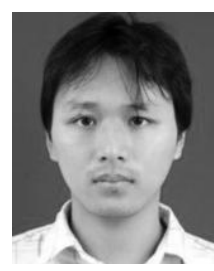

Jun Deng was born in Chongqing, China, in 1985.He received the B.S. and M.S. degrees in electrical engineering from Chongqing University, Chongqing, in 2007 and 2010, respectively. He is currently working toward the Ph.D. degree from the South China University of Technology, Guangzhou, China, where he is involved in the research of electromagnetic environment and system debugging.

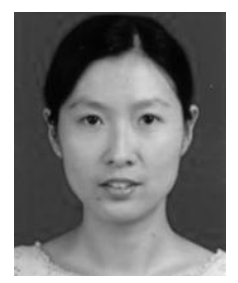

Yanpeng Hao received the B.S. and Ph.D. degrees in electrical engineering from Xi'an Jiaotong University, Xi'an, China, in 1998 and 2003, respectively. He is currently with the School of Electric Power, South China University of Technology, Guangzhou, China His research interests include insulation state diagnosis of key power equipments, the power system overvoltage and protection, and atmospheric pressure dielectric barrier discharge.

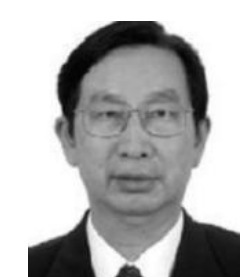

Licheng Li received the Chinese Academy of Engineering. He is currently the Experts Committee Secretary of China Southern Power Grid, Guangzhou, China. He is also with the School of Electric Power, South China University of Technology, Guangzhou. His research interests include HVDC key technology, outside the insulation for power system, transmission line online monitoring and fault warning, and overvoltage and power system protection 